\title{
Risks and benefits of reinduction ipilimumab/nivolumab in melanoma patients previously treated with ipilimumab/nivolumab
}

\author{
Paul B Chapman (D) , ${ }^{1}$ Vetri Sudar Jayaprakasam, ${ }^{2}$ Katherine S Panageas, ${ }^{3}$ \\ Margaret Callahan, ${ }^{1}$ Michael A Postow (D) , ${ }^{1}$ Alexander N Shoushtari (D) , ${ }^{1}$ \\ Jedd D Wolchok, ${ }^{1}$ Allison Betof Warner
}

To cite: Chapman PB, Jayaprakasam VS, Panageas KS, et al. Risks and benefits of reinduction ipilimumab/ nivolumab in melanoma patients previously treated with ipilimumab/nivolumab. Journal for ImmunoTherapy of Cancer 2021;9:e003395. doi:10.1136/ jitc-2021-003395

Accepted 14 September 2021

Check for updates

(C) Author(s) (or their employer(s)) 2021. Re-use permitted under CC BY-NC. No commercial re-use. See rights and permissions. Published by BMJ.

${ }^{1}$ Department of Medicine, Memorial Sloan Kettering Cancer Center, New York, New York, USA

${ }^{2}$ Department of Radiology,

Memorial Sloan Kettering Cancer Center, New York, New York, USA

${ }^{3}$ Department of Epidemiology and Biostatistics, Memorial Sloan Kettering Cancer Center, New York, New York, USA

Correspondence to

Dr Paul B Chapman;

chapmanp@mskcc.org

\section{ABSTRACT}

Background In melanoma patients who progress after prior ipilimumab/nivolumab (ipi/nivo) combination immunotherapy, there is no information regarding the risks and benefits of reinduction ipi/nivo.

Methods This was a retrospective review of 26 melanoma patients treated at Memorial Sloan Kettering Cancer Center (MSKCC) since 2012 who received reinduction ipi/nivo at least 6 months following completion of an initial course of ipi/nivo. We collected data on demographics, genetics, immune-related adverse events (irAEs), best overall responses (BORs), time to treatment failure (TTF) and overall survival (OS).

Results The BOR rate (complete response +partial response) was $74 \%$ (95\% Cl 52\% to $90 \%$ ) after the first course of ipi/nivo but only $23 \%$ (95\% Cl $8 \%$ to $45 \%)$ ) after reinduction. Response to reinduction did not correlate with response to the initial course. Among the 16 patients who had an objective response to the first course, only four $(25 \%)$ responded to reinduction. Of five patients who did not respond to the first course, one responded to reinduction. For all patients, median TTF was 5.3 months after reinduction; TTF was shorter for reinduction than for the first course in $85 \%$ of patients. Median OS from reinduction was 8.4 months; estimated 2-year OS was $18 \%$. Although reinduction was associated with fewer irAEs than the initial course of ipi/nivo ( $58 \%$ of patients vs $85 \%$ of patients in the initial course), eight ( $31 \%$ ) patients experienced at least one new irAE after the second course. Conclusions BOR rate and TTF were markedly less favorable after reinduction with ipi/nivo than after the initial course of ipi/nivo. Reinduction ipi/nivo was associated with frequent irAEs although less frequent than for the initial course.

\section{BACKGROUND}

Starting with the first phase 1 trial of ipilimumab/nivolumab (ipi/nivo) combination, ${ }^{1}$ multiple trials have confirmed the high level of antimelanoma activity. ${ }^{2-4}$ This combination has also become a treatment of choice for melanoma patients with brain metastases. ${ }^{56}$ Because of the mechanism of action and high response rate, ipi/nivo has been tested in patients with other malignancies and has shown impressive activity in lung cancer, ${ }^{7}$ renal cell carcinoma, ${ }^{8}$ and others. ${ }^{9}$ Ipi/nivo is associated with a high incidence of immunerelated adverse events (irAEs), most of which are serious and require treatment. Some irAEs do not resolve. This has led to ongoing discussions as to the risks and benefits of ipi/ nivo in various melanoma patient cohorts.

We have a long experience in our institution of using ipi/nivo in melanoma, and during this time, we have had the opportunity to re-treat melanoma patients who progressed following an initial course of ipi/nivo with a second (or reinduction) course of ipi/nivo; this was generally not allowed on the previous clinical trials. Reinduction with ipi monotherapy has been shown to be effective in a minority of patients after an initial course of ipi $^{10-12}$; similarly, reinduction with anti-PD1 therapy can benefit a minority of patients after an initial course of anti-PD1 therapy ${ }^{13}$ Little is known about the efficacy of reinduction ipi/nivo even though reinduction ipi/ nivo is listed in the NCCN guidelines ${ }^{14}$ as a treatment option. We describe this cohort of melanoma patients who were treated with reinduction ipi/nivo at least 6 months after the end of the first induction course of ipi/ nivo. In reporting the outcomes, we hope to provide guidance on the risks and benefits of reinduction ipi/nivo.

\section{METHODS}

\section{Patients and treatment}

We studied outcomes in patients who had received at least two separate courses of ipi/ nivo. To be a separate course, there had to be at least a 6-month interval between doses. 
We performed an electronic search of the electronic medical record at MSKCC for melanoma patients treated with ipi/nivo between January 2012 and December 2020. Specifically, we searched for patients who had received more than four doses of ipi/nivo or who had received two doses separated by at least 6 months. Patients who received maintenance anti-PD1 therapy after the initial course of ipi/nivo were included if there had been at least 6 months between the completion of maintenance anti-PD1 and the start of reinduction ipi/nivo. Cases were confirmed by detailed chart review. Basic demographics were collected including treatment dates, date of last follow-up and date of death. In patients in whom the melanoma had undergone next-generation sequencing of actionable cancer targets, ${ }^{15}$ we collected information on driver mutations, tumor mutational burden, and class I HLA identification.

irAEs and time-to-treatment failure (TTF), defined as the time from start of ipi/nivo to the start of a subsequent treatment (either systemic therapy, radiation therapy, or surgery but not including maintenance anti-PD1 therapy) or death, were collected by chart review. No attempt was made to grade the irAEs, but we did note if the patient required corticosteroids, infliximab, or mycophenolate immunosuppression for irAEs.

A retrospective radiographic review was performed in order to assess best overall response (BOR) according to RECIST V.1.1.

\section{Statistical analysis}

Patient and tumor characteristics were summarized using summary statistics. Overall survival was defined as the time from date of first (or second) course of ipi/nivo to date of death or date last known alive. TTF was defined as the time from the start of the first (or second) course of ipi/nivo to the date of the next treatment, death, or date last known alive. TTF from first cycle (y-axis) and reinduction (x-axis) were plotted on a correlation plot to visualize any associations between time to treatment failure after each treatment. McNemar's test was used for paired data to compare best objective response rate (complete response (CR)/partial response (PR) vs other) assessed after first course ipi/nivo and reinduction.

\section{RESULTS}

Our search initially identified 53 potential patients. After chart review, we eliminated 27 patients either because they did not have melanoma, had not received ipi/nivo, or had received multiple courses with no interruption of at least 6 months. The remaining 26 patients form the basis of this report.

\section{Patient demographics}

The 26 patients in this cohort (table 1) were typical of patients with metastatic melanoma. There were 17 men and 9 women with a median age of 57.6 years at the time of initial ipi/nivo treatment. The primary site was cutaneous
Table 1 Patient demographics $(n=26)$

\begin{tabular}{|c|c|c|}
\hline Age & Median (range) & $57.6(30.1-82.8)$ \\
\hline \multirow[t]{2}{*}{ Gender } & Male & 17 \\
\hline & Female & 9 \\
\hline \multirow{5}{*}{$\begin{array}{l}\text { American Joint } \\
\text { Committee on } \\
\text { Cancer stage }\end{array}$} & III & 4 \\
\hline & M1A & 0 \\
\hline & M1B & 3 \\
\hline & M1C & 11 \\
\hline & M1D & 7 \\
\hline \multirow[t]{4}{*}{ Primary site } & Cutaneous & 17 \\
\hline & Acral & 4 \\
\hline & Subungual & 1 \\
\hline & Unknown primary & 4 \\
\hline \multirow[t]{5}{*}{ Driver mutation } & BRAF & 10 \\
\hline & NRAS & 5 \\
\hline & Other* & 2 \\
\hline & No driver identified & 6 \\
\hline & Not tested & 3 \\
\hline \multirow{3}{*}{$\begin{array}{l}\text { Tumor mutation } \\
\text { burden }\end{array}$} & High & 11 \\
\hline & Low & 11 \\
\hline & Unknown & 4 \\
\hline \multirow{2}{*}{$\begin{array}{l}\text { First course of ipi/ } \\
\text { nivo }\end{array}$} & Median \# doses (range) & $4(1-4)$ \\
\hline & $\begin{array}{l}\text { \# of patients receiving } \\
\text { maintenance anti-PD1 }\end{array}$ & 16 \\
\hline $\begin{array}{l}\text { Reinduction } \\
\text { course of ipi/nivo }\end{array}$ & Median \# of doses (range) & $3(1-4)$ \\
\hline $\begin{array}{l}\text { Months between } \\
\text { courses }\end{array}$ & Median (range) & $22.2(6-49.1)$ \\
\hline
\end{tabular}

*One patient had KRAS G12V; the other had two NF1 alterations, X421_splice and Q392* and SF3B1 G740E.

ipi/nivo, ipilimumab/nivolumab.

in $65 \%$ of cases and was unknown in 4/26 patients (15\%). The remaining five patients $(27 \%)$ had acral or subungal primary sites. There were no patients with mucosal or choroidal primaries. At the time of initial ipi/nivo treatment, four patients had unresectable stage III melanoma; the remaining patients had stage IV melanoma including seven patients with central nervous system metastases (stage IVD).

The driver mutation was BRAFin 10 patients, NRAS in 5 patients, and 2 patients had other driver mutations (KRAS and NF1 inactivation, respectively). In six cases, we could not identify a driver mutation, and in three cases, genetic analysis was not performed. Half of the cases analyzed qualified as having a high mutational burden (defined as $\geq 10$ mutations/Mb). The patient's class I HLA-A, B, and $\mathrm{C}$ alleles were identified as well from this analysis. 
Table 2 Concordance of BOR between courses of ipi/nivo

BOR to reinduction course of ipi/ nivo (\# of patients)

\begin{tabular}{|c|c|c|c|c|c|}
\hline \multirow{5}{*}{$\begin{array}{l}\text { BOR to initial } \\
\text { course of ipi/ } \\
\text { nivo (\# of } \\
\text { patients) }\end{array}$} & & \multirow[t]{2}{*}{ CR } & PR & Stable & POD \\
\hline & CR & & & \multicolumn{2}{|l|}{1} \\
\hline & PR & \multirow[t]{3}{*}{2} & 2 & 6 & 5 \\
\hline & Stable & & 1 & 2 & 2 \\
\hline & POD & & & & \\
\hline
\end{tabular}

Table includes only the 21 patients who had measurable disease by RECIST prior to both courses of ipi/nivo. Blue colored squares indicate patients who had a poorer response to the reinduction course of ipi/nivo compared with the first course. The red colored squares indicate patients who had a better response to the reinduction course of ipi/nivo compared with the first.

BOR, best overall response; CR, complete response; ipi/nivo, ipilimumab/nivolumab; POD, progression; PR, partial response.

\section{Treatment courses}

Ipi/nivo was administered every 3 weeks using ipilimumab at dose of $3 \mathrm{mg} / \mathrm{kg}$ and nivolumab at $1 \mathrm{mg} / \mathrm{kg}$. In $3 / 150$ treatments (all during the reinduction course of ipi/ nivo), ipilimumab was used at $1 \mathrm{mg} / \mathrm{kg}$ and nivolumab at either $3 \mathrm{mg} / \mathrm{kg}$ or $240 \mathrm{mg}$ flat dose.

During the first course of ipi/nivo, the median number of doses was 4 (range 1-4); 16 patients $(61.5 \%)$ received maintenance nivolumab after the first course. The median time to reinduction with ipi/nivo was 22.2 months (range $6-49.1$ ). Nine patients (35\%) had received a total of 10 treatment regimens after induction ipi/nivo but prior to reinduction (four received BRAF/MEK inhibition; three received chemotherapy, two received ipi; one was treated on a phase I trial). For reinduction, the median number of ipi/nivo doses was 3 (range 1-4); seven patients received all four doses of reinduction ipi/nivo.

\section{Efficacy}

The BOR after the first course of ipi/nivo was a CR in 1 patient, $P R$ in 16 patients, and stable disease in 6 patients; three patients did not have tumor measurable by RECIST. This corresponds to a $74 \%$ (95\% CI $52 \%$ to $90 \%$ ) response rate. After reinduction with ipi/nivo, we observed two CRs, three PRs, nine stable disease, and eight progression of disease as BOR; four patients did not have measurable disease. This corresponded to only a $23 \%$ (95\% CI $8 \%$ to $45 \%$ ) response rate. We analyzed the concordance of response between the initial and the reinduction course of therapy at the individual patient level (table 2). Of the 21 patients who had measurable tumor prior to both courses of ipi/nivo, only three (14\%) had a better objective response after reinduction compared with the first course, while $14(67 \%)$ had a worse objective response to reinduction. Of the 16 evaluable patients who had a CR or PR to the first course of ipi/nivo, only four $(25 \%)$ responded to reinduction with ipi/nivo. Of
A

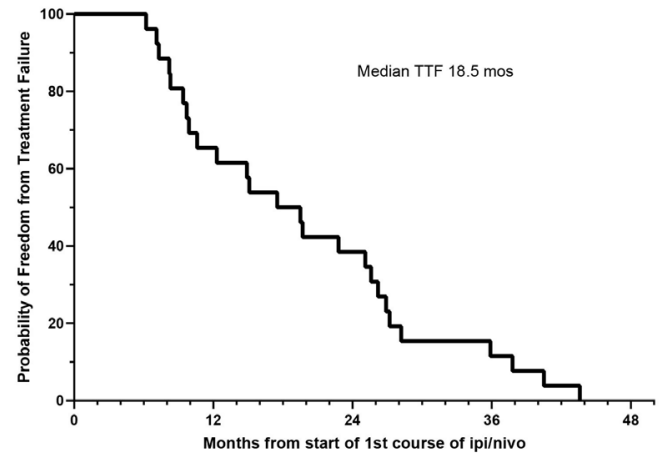

B

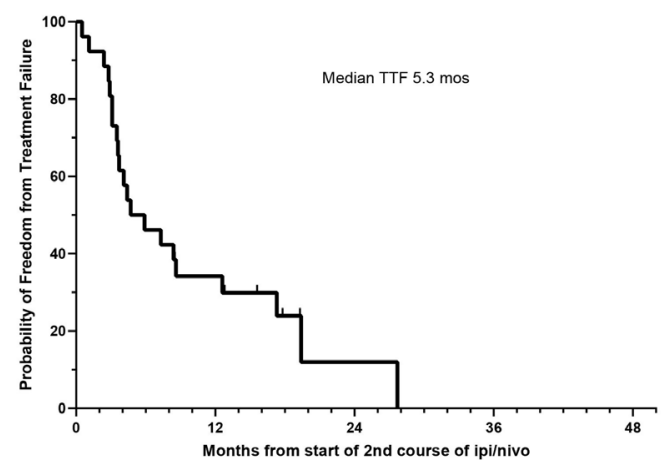

C

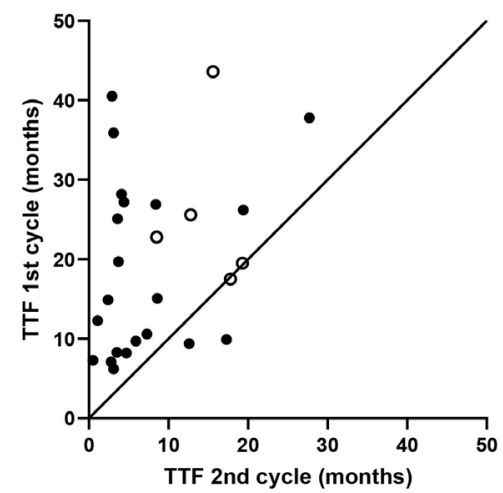

Figure 1 Time-to-treatment failure (TTF) for all patients from the start of the initial course if ipi/nivo (A) and from the start of reinduction ipi/nivo (B). Tick marks indicate censored patients. Figure part $\mathrm{C}$ compares the TTF seen after the initial course if ipi/nivo with the TTF seen after reinduction for each individual patient. Open circles indicate patients censored for TTF after reinduction with ipi/nivo. The equivalence line is indicated. ipi/nivo, ipilimumab/nivolumab. 
the five patients who had stable disease as BOR to the first course of ipi/nivo, only one responded to reinduction (PR that lasted 4.1 months). Initial response to the first course of ipi/nivo was not associated with response to re-induction (McNemar's test; $p$ value $=0.002$ ) .

By definition, all patients in this cohort progressed after the first course of ipi/nivo; the median TTF was 18.5 months (95\% CI 9.9 to 25.6) (figure 1A). However, after reinduction with ipi/nivo, the median TTF was only 5.3 (95\% CI 3.5 to 12.6) months (figure 1B). The estimated treatment failure rate after reinduction was $100 \%$ by 28 months. In comparing the TTF after the first course with the TTF of the reinduction course of ipi/nivo for each patient (figure 1B, the TTF after reinduction was shorter by a median of 8 months. At an individual patient level, 22/26 patients (85\%) experienced shorter TTF after reinduction compared with the first course (median: 12 months, range: 3-38 months). In two patients, TTF was the same after initial ipi/nivo and reinduction. In two patients, TTF after reinduction was slightly longer (3.2 months and 5.4 months) than for the first course.

The median overall survival from the start of the first course of ipi/nivo was 35.3 months (figure 2). The estimated survival at 4 years was $28 \%$. However, from the start of reinduction with ipi/nivo, the median survival was only

\section{A}
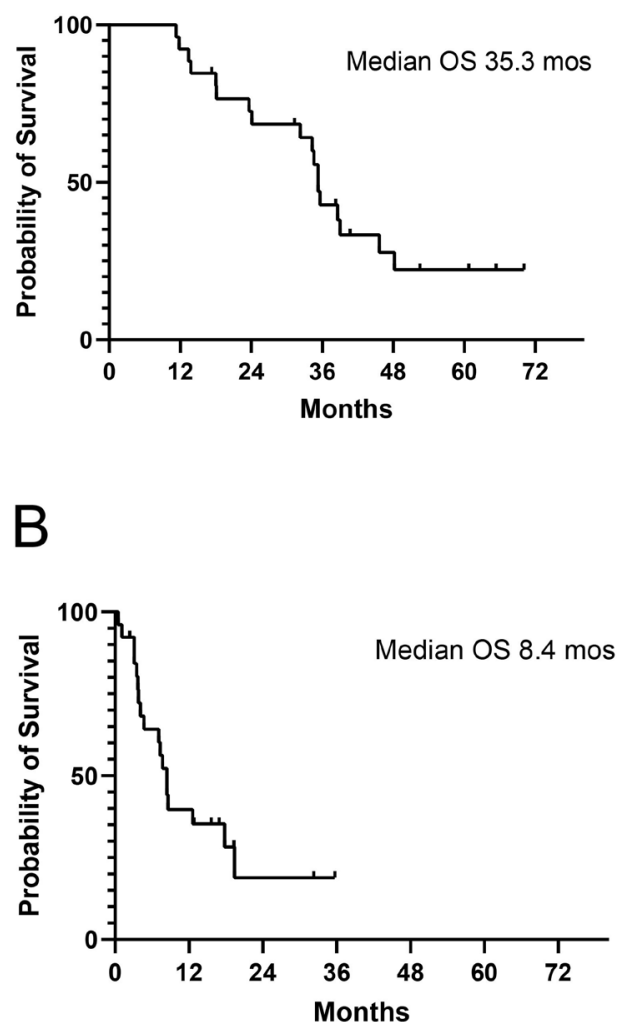

Figure 2 Overall survival (OS) from the start of the first course of ipi/nivo (A) and from the start of reinduction ipi/ nivo (B). Tick marks indicate censored patients. ipi/nivo, ipilimumab/nivolumab.
8.4 months; the estimated 2-year survival was only $19 \%$. This is consistent with the low ORR and TTF observed after reinduction with ipi/nivo.

We did not observe any correlation between treatment efficacy and driver mutation, tumor mutation burden, or class I HLA type.

\section{Immune-related adverse events}

The irAEs observed are indicated in table 3 . Twenty-two of 26 patients $(85 \%)$ had at least one irAE as a result of the first course of ipi/nivo; 13 (50\% of all patients) required treatment with infliximab and/or corticosteroids. The most common irAEs were hepatitis, rash, and colitis. After reinduction ipi/nivo, only $58 \%$ of patients experienced an irAE with the second course, but 11 patients required immunosuppression with corticosteroids, infliximab, or mycophenolate ( $42 \%$ of all patients). The most common irAEs as a result of the second course of treatment were again hepatitis, rash, and colitis. Only two patients received both courses of ipi/nivo without experiencing an irAE. The irAEs that most commonly occurred in both the initial and the reinduction course of ipi/nivo were: hepatitis, colitis, rash, and fever. Eight patients (31\%) experienced one or more serious irAEs after the reinduction course of therapy that they had not experienced after the first course including: type I diabetes, pneumonitis, hypopituitarism, meningitis, colitis, and hepatitis. We did not observe any correlation between incidence of irAE and class I HLA type.

\section{DISCUSSION}

In treatment-naïve patients, combination ipi/nivo is associated with $58 \%$ response rate with $22 \%$ CRs. ${ }^{16}$ This translated into 5-year progression-free survival of $48 \%$ in BRAF-mutated patients and $38 \%$ in BRAF wild-type patients. Five-year overall survival was $60 \%$ and $48 \%$, respectively. Based on these data and other supportive data, ${ }^{2-6}$ ipi/nivo has become a standard of care for the treatment of metastatic melanoma.

There are a variety of known and conjectured mechanisms of resistance to checkpoint inhibitor therapy, both native and acquired. ${ }^{17-21}$ The patient's T cell repertoire may not contain $\mathrm{T}$ cells capable of recognizing the specific tumor antigen expressed by the melanoma. Melanoma cells may downregulate expression of tumor antigens if the protein is not required for tumor cell viability. If the melanoma cell cannot downregulate the protein antigen, it can downregulate expression of HLA molecules responsible for presentation of the peptide antigen. The melanoma itself can acquire an immunosuppressive phenotype by loss of IFN $\gamma / \mathrm{JAK} / \mathrm{STAT}$ signaling or by producing inhibitory cytokines such as IL6 or IL1 $\beta$. The tumor microenvironment can be immunosuppressive due to regulatory $\mathrm{T}$ cells, suppressive macrophages, or myeloid-derived suppressive T cells. Given these mechanisms of acquired or native resistance, it is not surprising that once a patient develops resistance to checkpoint 
Table 3 Immune-related adverse events

\begin{tabular}{|c|c|c|c|}
\hline irAE & First course ipi/nivo & Second course ipi/nivo & Both courses \\
\hline Hepatitis & $00000 \bullet \bullet \bullet \bullet \bullet \bullet \bullet$ & $\infty \bullet \bullet \bullet$ & 00 \\
\hline Rash & ( $\bullet \bullet \bullet \bullet \bullet \bullet \bullet$ & $\bullet \bullet \bullet$ & $\bullet$ \\
\hline Fever & $\bullet \bullet \bullet \bullet$ & $\bullet \bullet$ & $\bullet$ \\
\hline Pancreatitis & $\bullet \bullet$ & $\bullet \bullet$ & \\
\hline Haemolytic anemia & (2) & (2) & (2) \\
\hline Sinusitis & & $\bullet$ & \\
\hline Meningitis & & • & \\
\hline Thyroid disorder & • & & \\
\hline Pneumonitis & & ( & \\
\hline Infusion reaction & - & & \\
\hline No toxicity & $\bullet \bullet \bullet \bullet$ & $\bullet \bullet \bullet \bullet \bullet \bullet \bullet \bullet \bullet \bullet \bullet$ & $\bullet \bullet$ \\
\hline
\end{tabular}

Each dot represents a patient. Dots surrounded by black circles indicate a toxicity that required corticosteroid therapy. Dots surrounded by red circles indicate a toxicity that was treated with either infliximab or mycophenolate. The last column indicates patients who experienced a toxicity in both courses of therapy. In this column, the dots are circled if infliximab or mycophenolate was required for either course of therapy. ipi/nivo, ipilimumab/nivolumab.

inhibitor treatment, even after an initial response, retreatment with checkpoint inhibitor therapy is usually less successful.

Experience with single-agent ipilimumab indicated that retreatment with ipilimumab could result in tumor responses ranging from $12 \%$ to $23 \% \cdot{ }^{10-12}$ Among patients treated with single-agent anti-PD1, retreatment with antiPD1 or ipi/nivo resulted in objective responses in $15 \%$ and $25 \%$ of patients, respectively. ${ }^{13}$ Recently, Olson ${ }^{22}$ and colleagues reported that in patients who had previously progressed on anti-PD1/PDL1 therapy, treatment with ipilimumab/pembrolizumab was associated with a $29 \%$ response rate. There is little published experience in reinduction with ipi/nivo among patients previously treated with ipi/nivo. Hepner et $a l^{23}$ described 47 patients who received ipi/nivo reinduction after progressing from prior ipi/nivo. Their cohort was slightly different than ours in that their patients all had experienced at least stable disease on induction ipi/nivo, and $15 \%$ of their patients were reinduced either with ipi alone or an attenuated ipi/anti-PD1 schedule. Despite these differences, the ORR and median PFS to reinduction were $26 \%$ and 5 months, similar to what we observed. Reoccurrence of the same irAEs was seen at reinduction in $40 \%$ of their patients.

In assessing the benefits of reinduction with ipi/nivo at least 6 months after completion of the first course of ipi/nivo, we found that $23 \%$ of patients achieved a CR or $\mathrm{PR}$ after reinduction. This is much lower than the BOR rate to the first cycle of ipi/nivo but very similar to the BOR rate seen in patients initially treated with either single agent ipilimumab or single agent anti-PD1 and then reinduced with the same agent, as noted previously. Objective response to the initial course of ipi/nivo was not associated with response to reinduction. The median TTF was only 5.3 months after reinduction, and in $85 \%$ of patients, responses to reinduction with ipi/nivo were shorter (by a median of 12 months) than the duration of response to the first cycle. Among the five patients with $\mathrm{CR} / \mathrm{PR}$ to reinduction ipi/nivo, the median TTF was 17.3 months, although two patients were censored for treatment failure at the time of data analysis. After reinduction, the overall survival after 1 year and 2 years was $40 \%$ and $19 \%$, respectively.

The irAEs associated with reinduction using ipi/nivo required immunosuppression in $58 \%$ of the patients and $31 \%$ of patients experienced a new irAE that had not been experienced during the first course. In two of these patients, the new irAE was irreversible (hypopituitarism and type I diabetes).

Overall, the risks and benefits of reinduction with ipi/ nivo are far less favorable than the risks and benefits to the initial course of ipi/nivo and resemble the risks and benefits of chemotherapy treatment for many malignancies: low response rates $(23 \%)$, few durable responses, high proportion of significant toxicity, and no clear evidence of prolonged survival. The objective response rate seen after reinduction with ipi/nivo in our cohort was similar 
to objective response rates seen in other studies after reinduction with either ipilimumab alone or anti-PD1 alone.

Going forward, we need to understand resistance mechanisms more fully and to ascertain whether any of them can be reversed or prevented. If resistance mechanisms are not reversible, we need new treatments that exploit different mechanisms of action. We should not anticipate the same high rate of object responses on ipi/nivo reinduction that we see with initial treatment with ipi/nivo, emphasizing the importance of clinical trials testing novel approaches for immunotherapy-resistant melanoma. In the meantime, in patients requiring reinduction therapy after ipi/nivo, it is reasonable to speculate from these data that we might decrease toxicity and achieve similar objective response rates using single-agent checkpoint inhibitors for reinduction.

\section{Twitter Allison Betof Warner @DrBetofMDPhD}

Contributors PC: original concept, data acquisition, and manuscript generation. VSJ: radiographic review and RECIST measurements. ABW: data analysis. KSP: data analysis and biostatistics. All coauthors reviewed the data and contributed to editing the manuscript.

Funding This work was funded in part by the Marie-Josée and Henry R. Kravis Center for Molecular Oncology and the National Cancer Institute Cancer Center Core Grant No. P30-CA008748. Some patients were treated on clinical trials funded in part by Bristol-Myers Squibb, the Ludwig Center for Cancer Research, or the Parker Institute for Cancer Immunotherapy.

Competing interests None declared.

Patient consent for publication Not applicable.

Ethics approval This retrospective analysis was approved by the Memorial Sloan Kettering Cancer Center institutional review board.

Provenance and peer review Not commissioned; externally peer reviewed.

Data availability statement Data are available on reasonable request. All data relevant to the study are included in the article or uploaded as supplementary information. We will make available a deidentified version of an Excel datasheet containing the extracted data.

Open access This is an open access article distributed in accordance with the Creative Commons Attribution Non Commercial (CC BY-NC 4.0) license, which permits others to distribute, remix, adapt, build upon this work non-commercially, and license their derivative works on different terms, provided the original work is properly cited, appropriate credit is given, any changes made indicated, and the use is non-commercial. See http://creativecommons.org/licenses/by-nc/4.0/.

\section{ORCID iDs}

Paul B Chapman http://orcid.org/0000-0003-1618-0811

Michael A Postow http://orcid.org/0000-0002-3367-7961

Alexander N Shoushtari http://orcid.org/0000-0002-8065-4412

Allison Betof Warner http://orcid.org/0000-0001-6422-2997

\section{REFERENCES}

1 Wolchok JD, Kluger H, Callahan MK, et al. Nivolumab plus ipilimumab in advanced melanoma. N Engl J Med 2013;369:122-33.
2 Postow MA, Chesney J, Pavlick AC. Nivolumab and ipilimumab versus ipilimumab in untreated melanoma. $N$ Engl $\mathrm{J}$ Med.

3 Larkin J, Chiarion-Sileni V, Gonzalez R. Combined nivolumab and ipilimumab or monotherapy in untreated melanoma. N Engl J Med 2015.

4 Hodi FS, Chapman PB, Sznol M, et al. Safety and efficacy of combination nivolumab plus ipilimumab in patients with advanced melanoma: results from a North American expanded access program (CheckMate 218). Melanoma Res 2021;31:67-75.

5 Tawbi HA-H, Forsyth PAJ, Algazi AP, et al. Efficacy and safety of nivolumab (NIVO) plus ipilimumab (IPI) in patients with melanoma (MEL) metastatic to the brain: results of the phase II study CheckMate 204. JCO 2017;35:9507-07.

6 Long GV, Atkinson V, Lo S, et al. Combination nivolumab and ipilimumab or nivolumab alone in melanoma brain metastases: a multicentre randomised phase 2 study. Lancet Oncol 2018;19:672-81.

7 Hellmann MD, Paz-Ares L, Bernabe Caro R, et al. Nivolumab plus ipilimumab in advanced Non-Small-Cell lung cancer. $N$ Engl $J$ Med 2019;381:2020-31.

8 Motzer RJ, Tannir NM, McDermott DF, et al. Nivolumab plus ipilimumab versus sunitinib in advanced renal-cell carcinoma. $N$ Engl J Med Overseas Ed 2018:378:1277-90.

9 McGregor BA, Campbell MT, Xie W, et al. Phase II study of nivolumab and ipilimumab for advanced bladder cancer of variant histologies (BCVH). JCO 2019;37:4518-18.

10 Lebbé C, Weber JS, Maio M, et al. Survival follow-up and ipilimumab retreatment of patients with advanced melanoma who received ipilimumab in prior phase II studies. Ann Oncol 2014;25:2277-84.

11 Robert C, Schadendorf D, Messina M, et al. Efficacy and safety of retreatment with ipilimumab in patients with pretreated advanced melanoma who progressed after initially achieving disease control. Clin Cancer Res 2013;19:2232-9.

12 Chiarion-Sileni V, Pigozzo J, Ascierto PA, et al. Ipilimumab retreatment in patients with pretreated advanced melanoma: the expanded access programme in Italy. Br J Cancer 2014;110:1721-6.

13 Betof Warner A, Palmer JS, Shoushtari AN, et al. Long-term outcomes and responses to retreatment in patients with melanoma treated with PD-1 blockade. J Clin Oncol 2020;38:1655-63.

14 Coit DG, Thompson JA, Albertini MR, et al. Cutaneous melanoma, version 2.2019, NCCN clinical practice guidelines in oncology. $J$ Natl Compr Canc Netw 2019;17:367-402.

15 Cheng DT, Mitchell TN, Zehir A, et al. Memorial Sloan Ketteringintegrated mutation profiling of actionable cancer targets (MSKIMPACT): a hybridization capture-based next-generation sequencing clinical assay for solid tumor molecular oncology. $J$ Mol Diagn 2015;17:251-64.

16 Larkin J, Chiarion-Sileni V, Gonzalez R, et al. Five-year survival with combined nivolumab and ipilimumab in advanced melanoma. $N$ Engl J Med 2019;381:1535-46.

17 D'Urso CM, Wang ZG, Cao Y, et al. Lack of HLA class I antigen expression by cultured melanoma cells FO-1 due to a defect in B2m gene expression. J Clin Invest 1991;87:284-92.

18 Restifo NP, Marincola FM, Kawakami Y, et al. Loss of functional beta 2-microglobulin in metastatic melanomas from five patients receiving immunotherapy. J Natl Cancer Inst 1996;88:100-8.

19 Zaretsky JM, Garcia-Diaz A, Shin DS, et al. Mutations associated with acquired resistance to PD-1 blockade in melanoma. $N$ Engl $J$ Med 2016;375:819-29.

20 Jenkins RW, Barbie DA, Flaherty KT. Mechanisms of resistance to immune checkpoint inhibitors. Br J Cancer 2018;118:9-16.

21 Sucker A, Zhao F, Real B, et al. Genetic evolution of T-cell resistance in the course of melanoma progression. Clin Cancer Res 2014;20:6593-604.

22 Olson DJ, Eroglu Z, Brockstein B. Pembrolizumab plus ipilimumab following Anti-PD-1/L1 failure in melanoma. JClinOncol.

23 Hepner A, Atkinson VG, Larkin J, et al. Re-induction ipilimumab following acquired resistance to combination ipilimumab and antiPD-1 therapy. Eur J Cancer 2021;153:213-22. 